\title{
Detection of Lung Dysfunction Using Ventilation and Perfusion SPECT in a Mouse Model of Chronic Cigarette Smoke Exposure
}

\author{
Brian N. Jobse ${ }^{1-3}$, Rod G. Rhem ${ }^{1}$, Iris Q. Wang ${ }^{1}$, William B. Counter ${ }^{1,3}$, Martin R. Stämpfli ${ }^{2,3}$, and N. Renée Labiris ${ }^{1,3}$ \\ ${ }^{I}$ Department of Medicine, Division of Respirology, McMaster University, Hamilton, Ontario, Canada; ${ }^{2}$ Department of Pathology and \\ Molecular Medicine, McMaster Immunology Research Centre, McMaster University, Hamilton, Ontario, Canada; and ${ }^{3}$ Firestone \\ Institute for Respiratory Health at St. Joseph's Healthcare, Hamilton, Ontario, Canada
}

Chronic obstructive pulmonary disease is a leading cause of morbidity and mortality worldwide. Exposure to cigarette smoke (CS) is a major risk factor for developing this chronic airflow impairment, but the early progression of disease is not well defined or understood. Ventilation/perfusion (V/Q) SPECT provides a noninvasive assessment of lung function to further our current understanding of how CS affects the lung. Methods: BALB/c mice were imaged with V/Q SPECT and CT after 8 and 24 wk of whole-body exposure to mainstream CS. Bronchoalveolar lavage was collected and cell differentials produced to determine inflammatory patterns. Histologic lung sections were collected, and a semiautomated quantitative analysis of airspace enlargement was applied to whole histology slices. Results: Exposure to CS induced an inflammatory response that included increases in the numbers of both mononuclear cells and neutrophils. Airspace enlargement was also significantly increased at 8 wk of CS exposure and was still more pronounced at $24 \mathrm{wk}$. Ventilation and perfusion correlation at the voxel level depicted a significant decrease in matching at $8 \mathrm{wk}$ of CS exposure that was also apparent after $24 \mathrm{wk}$. The standard deviation $(\mathrm{SD})$ of the $\log (\mathrm{V} / \mathrm{Q})$ curve, a basic measure of heterogeneity, was increased from $0.44 \pm 0.02$ in age-matched controls to $0.62 \pm 0.05$ with CS exposure at $24 \mathrm{wk}$, indicating an increase in V/Q mismatching between 8 and 24 wk of CS exposure. CT, however, was not capable of discriminating control from CS-exposed animals at either time point, even with greater resolution and respiratory gating. Conclusion: This study demonstrated that, before CT detection of structural changes, V/Q imaging detected changes in gas-exchange potential. This functional impairment corresponded to increased lung inflammation and increased airspace enlargement. In vivo V/Q imaging can detect early changes to the lung caused by CS exposure and thus provides a noninvasive method of longitudinally studying lung dysfunction in preclinical models. In the future, these measures could be applied clinically to study and diagnose the early stages of chronic obstructive pulmonary disease.

Received Jul. 17, 2012; revision accepted Oct. 10, 2012.

For correspondence or reprints contact: N. Renée Labiris, 1280 Main St. W., Health Sciences Centre, Room 1V11, Hamilton, Ontario, Canada L8S $4 \mathrm{~K} 1$.

E-mail: labir@mcmaster.ca

Published online Feb. 8, 2013

COPYRIGHT @ 2013 by the Society of Nuclear Medicine and Molecular Imaging, Inc.
Key Words: gas exchange; SPECT; histology; COPD; V/Q

J Nucl Med 2013; 54:616-623

DOI: 10.2967/jnumed.112.111419

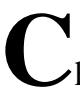

hronic obstructive pulmonary disease (COPD) is a worldwide health concern with high morbidity and mortality and an increasing burden on health care systems $(1,2)$. Exposure to cigarette smoke (CS), which is a major risk factor in COPD pathogenesis, leads to various pathologies including, but not limited to, chronic inflammation, increased mucus production, small-airway fibrosis, and airspace enlargement, otherwise known as emphysema (3). Together, these pathologies, which are heterogeneously present in the small airways and periphery of the lung, lead to a reduction in overall gas-exchange function that becomes debilitating over time (l). Traditionally, COPD is diagnosed by spirometric measures such as forced vital capacity and forced expiratory volume over $1 \mathrm{~s}$; these airflow-measurement techniques are also used in staging disease severity, monitoring disease progression, and measuring drug efficacy.

Although diagnosis of COPD by spirometry allows for a relatively simple measure of lung-function and diseaseseverity staging in afflicted individuals, the pathology of COPD tends to be in the smallest airways and these airways contribute little to airflow resistance under normal circumstances (4). Therefore, spirometry cannot detect the impact of small-airway obstruction until it has developed considerably, making spirometry insensitive to early disease. Further, spirometry does not differentiate the varying pathologies, such as small-airway obstruction and emphysema, associated with COPD $(5,6)$. Because of the lack of early diagnoses, due in part to the insensitivity of spirometry and the heterogeneous nature of the disease, a thorough understanding of early pathogenesis is still required. Until this disease can be phenotyped and precise roles of the various pathologies determined in relation to air flow, blood flow, and gas exchange, our inability to prevent lung dysfunction or provide patients with fully individualized treatment regimens will continue $(7,8)$. It is therefore necessary to develop tools that can be used to 
phenotype COPD, provide earlier diagnosis, and allow for greater understanding of this disease.

Clinical methods that measure ventilation/perfusion (V/Q) ratios in the lung have been shown to have high sensitivity to COPD pathologies, as demonstrated by Rodríguez-Roisin et al. using the multiple inert gas elimination technique (MIGET) (9). Although this methodology is well suited to understanding respiratory disease mechanisms because of its ability to describe the distribution of V/Q ratios in the lung, it lacks the ability to spatially distinguish underlying pathologies (10) and is not readily available in clinical practice. V/Q SPECT imaging, on the other hand, is a well-established nuclear medicine technique that provides spatial information regarding the 2 core processes of respiratory gas exchange, ventilation of alveolar units and perfusion of the pulmonary capillary beds (11), and the results of this technique correlate well with those derived from MIGET (12). SPECT V/Q is commonly used in the diagnosis of pulmonary embolism but has been shown to be sensitive to early indicators of COPD in addition to being capable of identifying comorbid disease and distinguishing pathophysiologic changes $(13,14)$. Furthermore, coregistration of nuclear imaging data to CT images allows for greater insight into the anatomic position of lung dysfunction and structural information such as the distribution of emphysema.

Although V/Q imaging techniques hold great promise in providing a better understanding of chronic respiratory illnesses such as COPD, these techniques can also be used in preclinical research to study disease pathogenesis and evaluate the efficacy of therapies. Preclinical models for investigating COPD include a host of different agents to elicit pathologies such as inflammation and emphysema, but CS exposure is likely the most relevant when modeling the pathogenesis of this disease (15).

We have applied V/Q imaging to a preclinical model of COPD to determine the impact of prolonged CS exposure on the relationship between ventilation and perfusion in the lung. Inflammatory measures and histologic assessment of airspace enlargement were collected to describe 2 of the major pathologies present in this model and to provide context for the V/Q measurements. In addition, anatomic data from CT images were compared with the functional measures obtained through V/Q imaging. Although CT may not be sensitive to early changes in the lung associated with CS exposure, V/Q imaging provides a method by which to study the functional consequences of the inflammation and airspace enlargement present under these conditions.

\section{MATERIALS AND METHODS}

\section{Animals}

Female BALB/c mice (Charles River) were acclimatized to specific pathogen-free housing conditions for 2-3 wk, with a 12-h-12-h lightdark cycle, before experimentation. The studies described here were approved by the Animal Research Ethics Board of McMaster University (Hamilton, Ontario, Canada) in accordance with the guidelines of the Canadian Council on Animal Care. Baseline and 24-wk control V/Q and CT data were published previously in a related article (16).

\section{CS Exposure Protocol}

The mice were exposed to the smoke generated from twelve 2R4F reference cigarettes (University of Kentucky), with the filters removed, for $50 \mathrm{~min}$ twice daily, $5 \mathrm{~d} / \mathrm{wk}$, using a SIU48 whole-body exposure system (Promech Laboratories) for either 8 or $24 \mathrm{wk}$. Details of the exposure protocol were reported previously (17). Average total particulate matter during exposure was $786 \mu \mathrm{g} / \mathrm{L}$. Control animals were exposed to room air alone. Before CS exposure, the animals were acclimatized to the system for $3 \mathrm{~d}$. Smoke exposure in this system is well tolerated and results in increased levels of carboxyhemoglobin and cotinine (17).

\section{Collection and Measurement of Specimens}

Approximately $16 \mathrm{~h}$ after the final CS exposure, the mice were sacrificed and bronchoalveolar lavage (BAL) was collected. The lungs were removed and lavaged twice with phosphate-buffered saline $(0.25 \mathrm{~mL}$ followed by $0.20 \mathrm{~mL})$. Total cell counts were determined using a hemocytometer. After centrifugation, the cell pellets were resuspended in phosphate-buffered saline, and slides were prepared by cytocentrifugation (Shandon Inc.) at $9.66 \mathrm{~g}$ for $2 \mathrm{~min}$. Hema3 (Fisher Scientific Co.) was used to stain all slides. Differential counts of BAL cells were determined from at least 500 leukocytes using standard hemocytologic procedures to classify the cells as neutrophils, eosinophils, or mononuclear cells. The number of animals in each group for cellular outcomes was as follows: 5 in the baseline group, 7 in the 8-wk control group, 8 in the 8 -wk CS group, 4 in the 24-wk control, and 7 in the 24-wk CS group. The left lung lobe was inflated to a pressure of $30 \mathrm{~cm}$ using $10 \%$ formalin, fixed, sectioned into 4 pieces, and embedded in paraffin; 3-mm-thick sections were stained with hematoxylin and eosin (H\&E). Images of whole H\&E-stained lung slices were acquired at $\times 16$ magnification and an image resolution of 2,560 $\times$ 1,920 pixels, producing pixels $6.098 \mu \mathrm{m}$ in length, on a DMRA microscope (Leica Microsystems). Airspace enlargement was quantified using Pneumometrics (version 1; Pneumometrics Software); each data point for an animal represents the average of 4 whole histologic slices obtained for the left lung lobe, with major airways and blood vessels identified by the user and removed. Briefly, the program converted the histologic image to a binary image and separated contiguous airspaces using a stepwise morphologic closing operation. The program then automatically measured the dimensions and perimeters of airspaces and provided the total area within a range of airspace sizes. The number of animals in each group for histologic outcomes was as follows: 5 in the baseline group, 7 in the 8-wk control group, 7 in the 8-wk CS group, 4 in the 24 -wk control group, and 6 in the 24-wk CS group.

\section{Imaging Protocol}

Imaging was performed as previously described (16). In brief, the mice were tracheally intubated through the oral cavity after anesthetization with ketamine and xylazine (90 and $6 \mathrm{mg} / \mathrm{kg}$, respectively). The animals were then ventilated $(0.02 \mathrm{~L} / \mathrm{min}, 125$ strokes/min) on a rodent ventilator (model 683; Harvard Apparatus) with Technegas (Cyclomedica) $(0.04-0.12 \mathrm{MBq} / \mathrm{mL})$ for $15 \mathrm{~min}$. After Technegas delivery, the animals were removed from the ventilator and allowed to breathe freely. SPECT scans were acquired on an X-SPECT system (Gamma Medica) using pinhole collimators and a radius of rotation of $3.5 \mathrm{~cm}$. A high-quality ventilation CT scan was collected immediately after the SPECT scan, also acquired on the X-SPECT system, with X-ray tube characteristics of $75 \mathrm{kVp}$ and $220 \mu \mathrm{A}$. After the ventilation SPECT and 
CT scans, the mice were injected with $11-15 \mathrm{MBq}$ of ${ }^{99 \mathrm{~m}} \mathrm{Tc}-\mathrm{mac}-$ roaggregated albumin via the tail vein. Perfusion imaging entailed a CT and SPECT scan. Supplemental gaseous anesthesia was used (isoflurane, $1 \%, 1 \mathrm{~L} / \mathrm{min}$ ) to ensure sedation throughout the imaging procedure.

SPECT and CT images were reconstructed, fused, and coregistered as previously described (16). Calibration of each CT image for Hounsfield unit (HU) scaling was performed using a waterfilled tube included in each scan and air within the field of view but external to the animal. A lung region of interest (ROI) was produced for the ventilation CT images, as described previously, using Amira 5.1 software (Visage Imaging). An example of an axial slice of the lung ROI is shown in Figure 1A. In-house-developed software was used to fuse the perfusion CT and SPECT images by a process that maximized mutual information through rigid-body transformation within the lung region as defined by the lung ROI, as previously described (16). Mutual information-derived parameters obtained from fusion of the perfusion SPECT and CT images

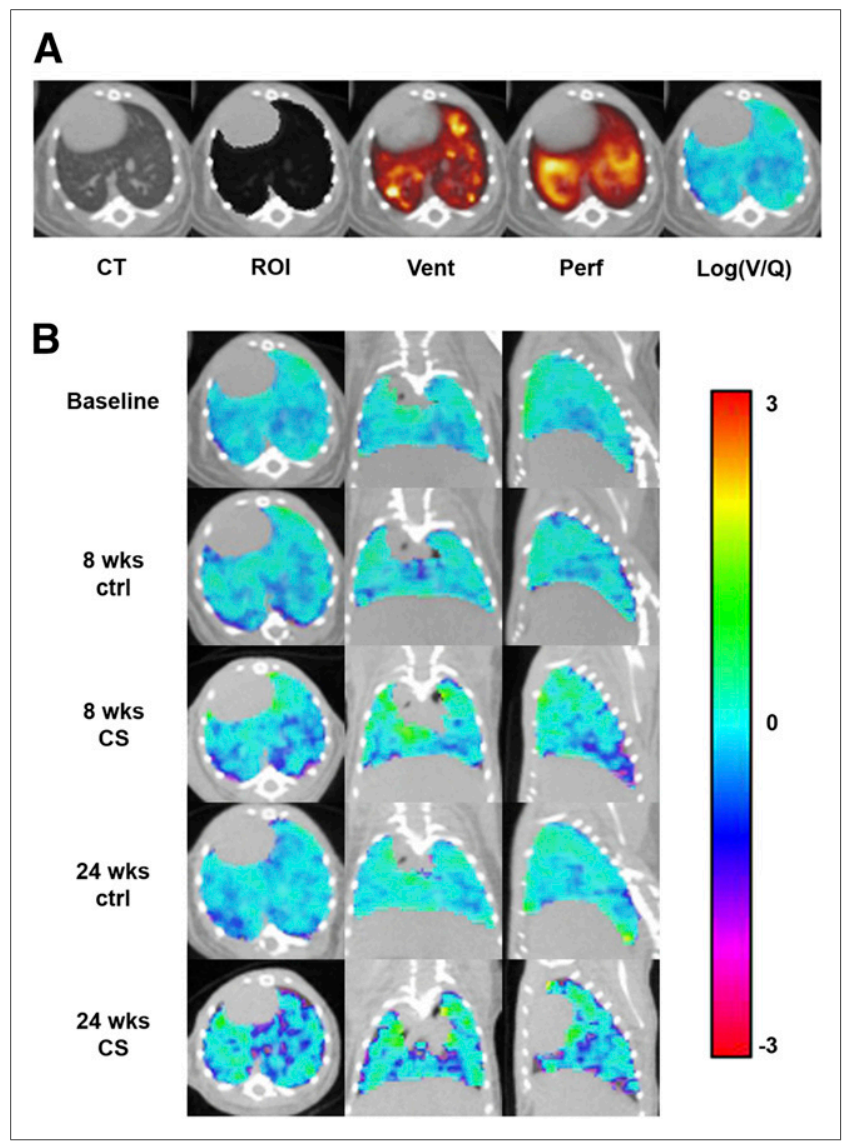

FIGURE 1. Representative SPECT and CT images. (A) CT and SPECT axial slice images for animal at baseline. (B) Log(V/Q) images in axial, coronal, and sagittal planes for all experimental time points. Animals shown were chosen on basis of average SD, mean, and ventilation-vs.-perfusion correlation values for dataset. Color bar indicates values seen in associated log(V/Q) images. CS = cigarette smoke exposure; $\mathrm{CT}=$ ventilation-associated $\mathrm{CT}$; $\mathrm{Ctrl}=$ agematched controls; $\log (\mathrm{V} / \mathrm{Q})=$ final calculated data; $\mathrm{ROI}=$ lung region of interest seen as black overlay on CT image; Vent and Perf $=$ ventilation and perfusion SPECT images, respectively, with color scale increasing from red through yellow to white for both. were then applied to the ventilation SPECT and CT images. The ventilation and perfusion datasets were then coregistered by repeating the mutual information maximization process through rigid-body transformation of their respective CT images, again within the lung segmentation.

\section{Respiration-Gated CT Image Processing and Quantitation}

Inspiration- and expiration-gated CT images were produced by applying RespGate software (RespGate) to all ventilation CT projections (18). Respiration-gated images were then reconstructed and calibrated as described for the ungated image used in the V/Q analysis. Lung-associated air volume from respiration-gated CT images was extracted by a method previously described based on a segmentation of the entire thoracic cavity $(16,19)$. Functional residual capacity and tidal volume were calculated from these data. To detect emphysematous changes, thresholds of -450 and $-550 \mathrm{HU}$ were used because these values are indicative of severe alveolar destruction in mouse models with the CT system used in this study (20).

\section{Quantitative Per-Voxel Image Data Analysis}

The coregistration of images allows for analysis of SPECT and CT data on a per-voxel basis. Within the lung ROI, all voxels have a density value (HU), relative ventilation and perfusion counts, V/Q, and a $\log (\mathrm{V} / \mathrm{Q})$ value (Fig. 1A); presented data represent mean distributions of all voxels within the lung ROI for each experimental group, unless otherwise stated. Ventilation and perfusion values were converted to relative frequencies by dividing the activity value of each voxel by the total activity in the lung ROI. The relationship between ventilation and perfusion data was determined by finding the Pearson correlation coefficient for the plot of the relative ventilation-versus-perfusion values in each voxel. V/Q ratios were calculated using the relative frequency of both ventilation and perfusion, and a base 10 logarithm was applied to provide a lognormal distribution. Analysis of the $\log (\mathrm{V} / \mathrm{Q})$ data involved calculation of the mean, standard deviation (SD), and percentage of total lung volume greater than \pm 2 averaged SDs from the averaged baseline mean $\log (\mathrm{V} / \mathrm{Q})$, as a measure of V/Q mismatching. Low $\log (\mathrm{V} / \mathrm{Q})$ henceforth refers to negative values greater than 2 SDs from the baseline mean, and high $\log (\mathrm{V} / \mathrm{Q})$ refers to positive values found by the same process. Because of the use of the logarithmic scale, any voxel for which only ventilation equaled zero was set to a $\log (\mathrm{V} / \mathrm{Q})$ ratio of $-\infty$ and any voxel for which only perfusion equaled zero was set to a $\log (\mathrm{V} / \mathrm{Q})$ ratio of $+\infty$. These values were not included in the calculation of the $\log (\mathrm{V} / \mathrm{Q})$ mean and SD but were included in measures of high and $\operatorname{low} \log (\mathrm{V} / \mathrm{Q})$. Any voxels for which both ventilation and perfusion equaled zero were noted and given a $\log (\mathrm{V} / \mathrm{Q})$ value of zero. The number of animals in each group for imaging outcomes was as follows: 5 in the baseline group, 4 in the 8-wk control group, 7 in the 8-wk CS group, 6 in the 24-wk control group, and 7 in the 24-wk CS group.

\section{Data Analysis}

Data were expressed as the mean \pm SEM. When age-matched experimental groups were compared, statistical significance was determined by unpaired, 2-tailed $t$ testing (GraphPad Software Inc.). Within an experimental group, results were compared between exposure time points and baseline using a 1-way ANOVA with Tukey 


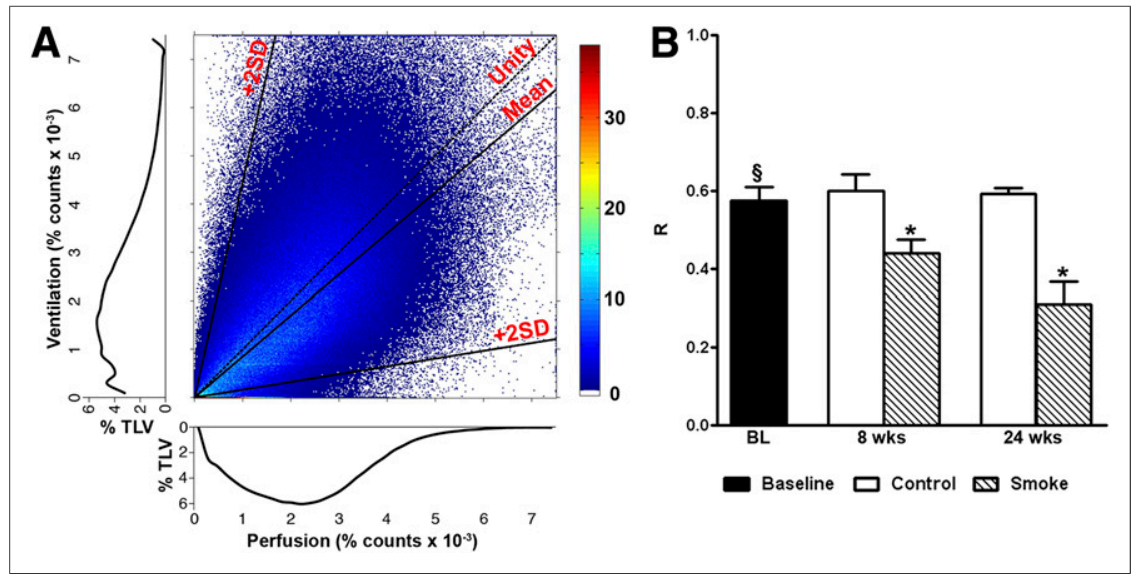

FIGURE 2. Relationship of ventilation and perfusion distributions. (A) Baseline ventilation-vs.-perfusion scatterplot representing averaged data. $x$ - and $y$-axes represent perfusion and ventilation, respectively; each point represents ventilation and perfusion value of a single voxel, and scale bar represents number of stacked voxels. Average baseline distributions for ventilation and perfusion are shown separately in axis-associated graphs. Line for $V=Q$ (unity), as well as lines for mean $\log (\mathrm{V} / \mathrm{Q})$ and 2 SDs from the mean, are shown. (B) Pearson correlation coefficient $(R)$ for assessing relationship between ventilation and perfusion counts. ${ }^{*} P<0.05$ CS vs. age-matched control by 2-tailed unpaired $t$ test. $\S P<0.05$ baseline vs. 24 wk CS by 1 -way ANOVA with Tukey post hoc. BL $=$ baseline.

post hoc analysis. In addition, 2-way ANOVA was applied to all measurements to test for interaction between smoke exposure and time. A $P$ value of less than 0.05 was considered statistically significant for all tests.

\section{RESULTS}

$\mathrm{BALB} / \mathrm{c}$ mice were exposed to $\mathrm{CS}$ or, to provide agematched controls, room air for a period of $24 \mathrm{wk}$. V/Q SPECT/CT images were acquired after 8 and 24 wk of CS exposure. Visual inspection of $\log (\mathrm{V} / \mathrm{Q})$ data indicated increased heterogeneity and mismatching over time in the CS-exposed groups as compared with their agematched controls (Fig. 1B). This mismatching was obvious after $24 \mathrm{wk}$; however, no specific patterns of mismatching were observed between animals within the CS-exposed groups.

To further evaluate ventilation and perfusion distributions, their association on a per-voxel basis was examined (Fig. 2A). The average ventilation-versus-perfusion distribution at baseline had a proportion of voxels falling on the line of unity, indicating perfectly matched ventilation and perfusion values. Although this situation was largely unchanged in age-matched controls, CS-exposed animals eventually lost this feature. As a quantification of these data, the correlation between ventilation and perfusion, measured by the Pearson coefficient, did not change over time in control groups but was significantly less in CS-exposed groups than in their age-matched controls at both 8 and 24 wk (Fig. 2B). These data indicate that V/Q mismatching caused by CS exposure was present as early as $8 \mathrm{wk}$.

V/Q relationships were further qualified through the distribution of $\log (\mathrm{V} / \mathrm{Q})$ values, for which a decrease in the peak and a widening of the curve were apparent with increasing CS exposure (Fig. 3A). The mean $\log (\mathrm{V} / \mathrm{Q})$ value of the 24-wk CS-exposure group was significantly more negative than that at baseline, in the 8-wk CS group, and in the age-matched controls at $24 \mathrm{wk}$ (Fig. 3B); a significant interaction in the mean $\log (\mathrm{V} / \mathrm{Q})$ was found between time and CS exposure through 2-way ANOVA. The SD of the $\log (\mathrm{V} / \mathrm{Q})$ distribution, a measure of heterogeneity,

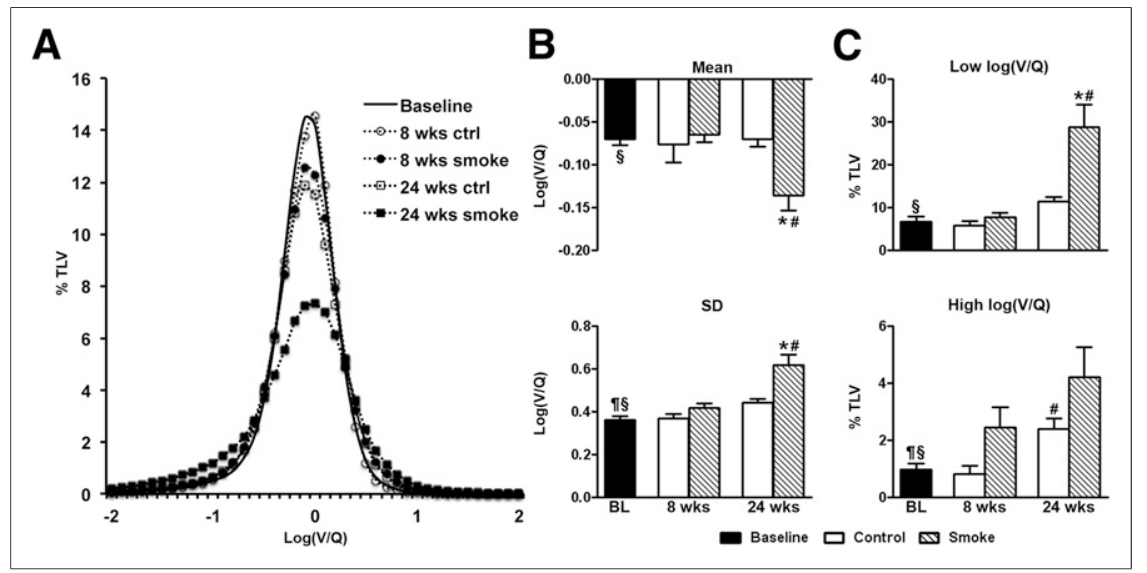

FIGURE 3. Log(V/Q) distribution. (A) Volumestandardized distribution of $\log (\mathrm{V} / \mathrm{Q})$ values measured in percentage of total lung volume (\%TLV). Baseline (black), 8-wk control $\bigcirc$, 8-wk CS-exposed $\bullet$, 24-wk control $\square$, and 24-wk CS-exposed $\mathbf{m}$ are shown. (B) Mean (top) and standard deviation (bottom) of $\log (\mathrm{V} / \mathrm{Q})$ distributions for all experimental groups, calculated without $\pm \infty$ values. (C) Percentage of total lung volume with log (V/Q) mismatching. Mismatching was defined as values greater or less than 2 average SDs from the average mean and include voxels where $\mathrm{V}=0(-\infty)$ and $\mathrm{Q}=0(+\infty)$. ${ }^{\star} P<0.05$ for CS vs. age-matched control by 2 -tailed unpaired $t$ test. ${ }^{\#} P<0.05$ for 24-wk control or CS vs. 8-wk control or CS by 1-way ANOVA with Tukey post hoc. ${ }^{\Uparrow} P<0.05$ for baseline vs. 24-wk control by 1 -way ANOVA with Tukey post hoc. $\$ P<0.05$ for baseline vs. 24 -wk CS by 1 -way ANOVA with Tukey post hoc. \%TLV = percentage of total lung volume; $\mathrm{BL}=$ baseline; $\mathrm{ctrl}=$ control. 
FIGURE 4. Inflammatory cell counts from BAL. Total cell number (A), mononuclear cells $(B)$, and neutrophils $(C)$ are shown. ${ }^{\star} P<0.05$ for CS vs. age-matched control by 2 -tailed unpaired $t$ test. $¥ P<0.05$ for baseline vs. 8- and 24-wk CS by 1-way ANOVA with Tukey post hoc. ${ }^{\#} P 0.05$ for 24-wk CS vs. 8-wk control or CS by 1-way ANOVA with Tukey post hoc. $\mathrm{BL}=$ baseline.

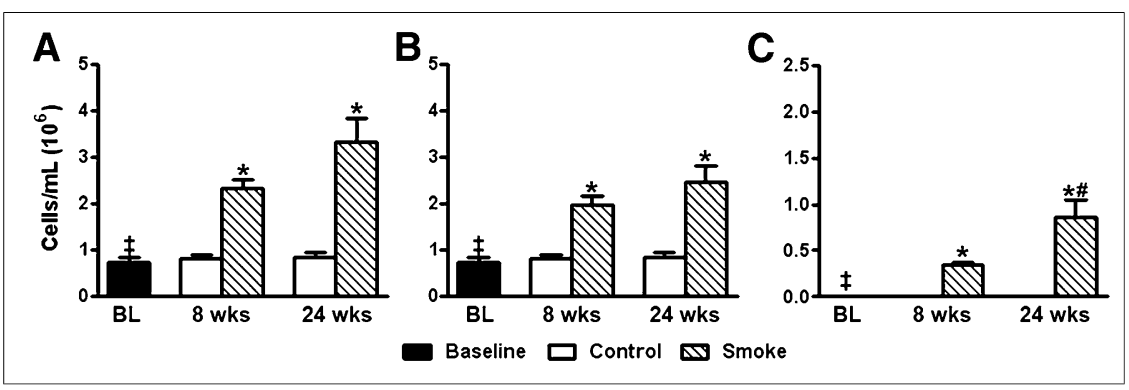

demonstrated no significant difference after 8 wk of CS exposure when compared with baseline and age-matched controls. After 24 wk of CS exposure, however, a significant increase in SD was found, compared with not only agematched controls but also baseline and 8-wk CS exposure. As reported previously (16), the age-matched control group at $24 \mathrm{wk}$ also demonstrated a significant increase in the SD of $\log (\mathrm{V} / \mathrm{Q})$ over that of baseline animals (Fig. 3B). Therefore, while V/Q mismatch is progressive with age there is still a significant increase in mismatching in CS-exposed mice over controls at $24 \mathrm{wk}$.

The percentage of lung volume with $\operatorname{low} \log (\mathrm{V} / \mathrm{Q})$ values - that is, reduced ventilation in comparison to perfusion-was substantially increased in the group with $24 \mathrm{wk}$ of CS exposure, compared with the age-matched control, baseline, and 8-wk CS groups (Fig. 3C). Further, there was a significant interaction between time and CS exposure in these low $\log (\mathrm{V} / \mathrm{Q})$ values. The proportion of high $\log (\mathrm{V} / \mathrm{Q})$ values, or voxels with greater ventilation than perfusion, was increased in both the CS-exposed mice and the controls at 24 wk, compared with baseline. In this model, the proportion of $\operatorname{low} \log (\mathrm{V} / \mathrm{Q})$ values was much greater than that of high $\log$ $(\mathrm{V} / \mathrm{Q})$ values.

BAL samples were collected to provide insight into the inflammatory environment associated with the V/Q mismatching observed. A significant increase in total leukocyte number in the BAL was found at $8 \mathrm{wk}$ for mice exposed to CS, compared with controls, and this inflammatory burden remained elevated up to 24 wk (Fig. 4). The absolute number of neutrophils was also significantly increased by $8 \mathrm{wk}$ of CS exposure, compared with age-matched controls, and a further increase in neutrophils was seen after 24 wk of exposure, compared with levels at $8 \mathrm{wk}$. The difference between 8 and 24 wk also reflects a significant interaction between time and CS exposure.

Airspace enlargement was measured in whole histologic H\&E-stained lung slices to determine the structural consequences of CS exposure. Representative histology slices from control and CS-exposed groups at 24 wk depict the overall extent of airspace enlargement (Fig. 5A). Quantification of all airspaces within the histologic images revealed that their size distribution was not gaussian. Therefore, airspace enlargement was analyzed by plotting the percentage of total airspace area against logarithmic ranges of airspace size to produce a simplified distribution. In animals exposed to 8 wk of CS, the area associated with larger airspaces, between $10^{4}$ and $10^{5} \mu \mathrm{m}^{2}$, was significantly increased over that of the age-matched controls. Animals exposed to $24 \mathrm{wk}$ of CS demonstrated a similar increase between $10^{4}$ and $10^{5}$

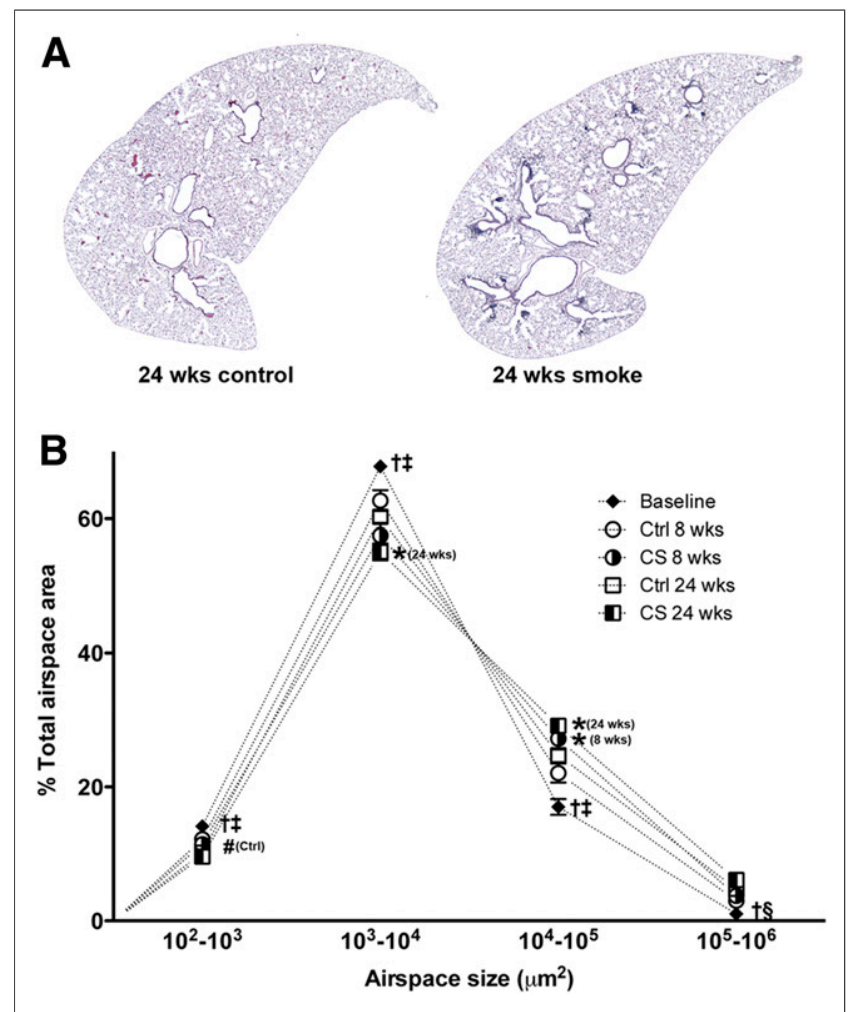

FIGURE 5. Assessment of airspace enlargement using quantitative histologic analysis. (A) Representative whole-lung slices, stained with $\mathrm{H} \& \mathrm{E}$, for control (left) and CS-exposed (right) mice at 24 wks. (B) Comparison of total airspace area associated with logarithmic airspace size ranges; connected points represent distribution of airspace area derived from whole-slice histologic quantification and averaged across animals. Statistical analyses were confined to airspace size ranges. Bracketed terms denote group to which statistical mark belongs. Major airways and vessels were removed before quantitation. ${ }^{*} P<0.05$ for $\mathrm{CS}$ vs. age-matched control by 2 -tailed unpaired $t$ test. ${ }^{\prime} P<0.05$ for 24 -wk control or CS vs. 8-wk control or CS by 1-way ANOVA with Tukey post hoc. $\$ P<0.05$ for baseline vs. 24-wk control by 1-way ANOVA with Tukey post hoc. ${ }^{\dagger} P<0.05$ for baseline vs. 8- and 24-wk control by 1-way ANOVA with Tukey post hoc. $\ddagger P<0.05$ for baseline vs. 8 - and 24 -wk CS by 1 -way ANOVA with Tukey post hoc. Ctrl $=$ control. 
$\mu \mathrm{m}^{2}$ and showed a significant reciprocal decrease in airspaces between $10^{3}$ and $10^{4} \mu \mathrm{m}^{2}$ (Fig. 5B). Increases in airspace enlargement were also observed in control animals in an age-dependent manner. Therefore, whereas a degree of airspace enlargement was observed with age alone, exposure to CS led to a significant increase in this pathology at both 8 and 24 wk.

In contrast, CT densitometry was not different between the 24-wk CS group and the age-matched control groups (Fig. 6). Summation of air volumes in the lung yielded no significant differences in functional residual capacity or tidal volume between CS groups and their age-matched controls (data not shown); however, a significant increase in functional residual capacity was observed between baseline and both controls and CS-exposed mice at 24 wk. Further, no difference was observed in the frequency of voxels less than $-550 \mathrm{HU}$ or $-400 \mathrm{HU}$ (data not shown) between CS groups and their controls, indicating no major volumes of emphysema were present.

\section{DISCUSSION}

Continuing exposure to CS is known to have immunologic and structural consequences (6) that eventually lead to a reduction in gas exchange capability. The progression of this lung dysfunction and how the core pulmonary processes of ventilation and perfusion are affected are issues that are not well defined. The ability of this methodology, and other similar 3-dimensional methods, to spatially examine the impact of varying pathologies on gas exchange in the lung provides the necessary tools to study complex and chronic diseases such as COPD. Preclinical investigation of ventilation and perfusion in models of COPD will allow for a better understanding of clinical disease progression by providing a translational approach to measurement of these core lung processes.

The approach taken in this study was to determine the impact of sustained CS exposure on the lung and the ability to match ventilation to perfusion in this context. Previously in clinical studies, Jogi et al. have demonstrated that V/Q SPECT is sensitive to COPD-related pathologies (13), and Suga et al. have quantified the V/Q mismatch due to emphysema in a per-voxel manner (14). Our results affirm that V/Q methodologies, being functional measurements, are sensitive to early pathologic changes that lead to COPD but may not be apparent, even at a higher resolution, with anatomic imaging systems such as CT. Because V/Q imaging is a common nuclear medicine technique and SPECT/CT systems are increasingly used (21), V/Q measurement could play an important role in the diagnosis, treatment, and further understanding of COPD $(13,22,23)$.

We have demonstrated that V/Q mismatching progressively increased during CS exposure in mice. The SPECT results presented here indicated that low V/Q mismatch predominated over high V/Q mismatch in the lungs of mice exposed to 24 wk of CS. The CS exposure model caused significant neutrophilia and monocyte infiltration whereas only a slight increase in airspace enlargement was observed after 24 wk. The V/Q profile observed in this context is consistent with clinical MIGET data in which V/Q mismatch was observed in GOLD stage I (9). MIGET studies have also provided evidence that high V/Q values tend to be present in patients with emphysematous pathology whereas low V/Q values tend to be present in patients with greater inflammatory indices and small-airway pathology $(24,25)$. Lower V/Q values have also been observed through PET after acute exposure to CS in sheep (26). The dominance of low V/Q mismatching in our model is therefore most likely associated with airway narrowing and obstruction caused by the increase in lung inflammation.

Although the effects of airspace enlargement in this model were small, a significant increase in airspace area was found in the size range between $10^{4}$ and $10^{5} \mu \mathrm{m}^{2}$, indicating that the effect was predominantly associated with larger airspaces in the acinar complex. The impact of age on the lung structure was also apparent between baseline and the control groups at either time point, as shown in Figure 5B. This result lends strength to findings derived from CT images in which an increase in volume was observed over baseline values in both CS-exposed and control mice but no differ-

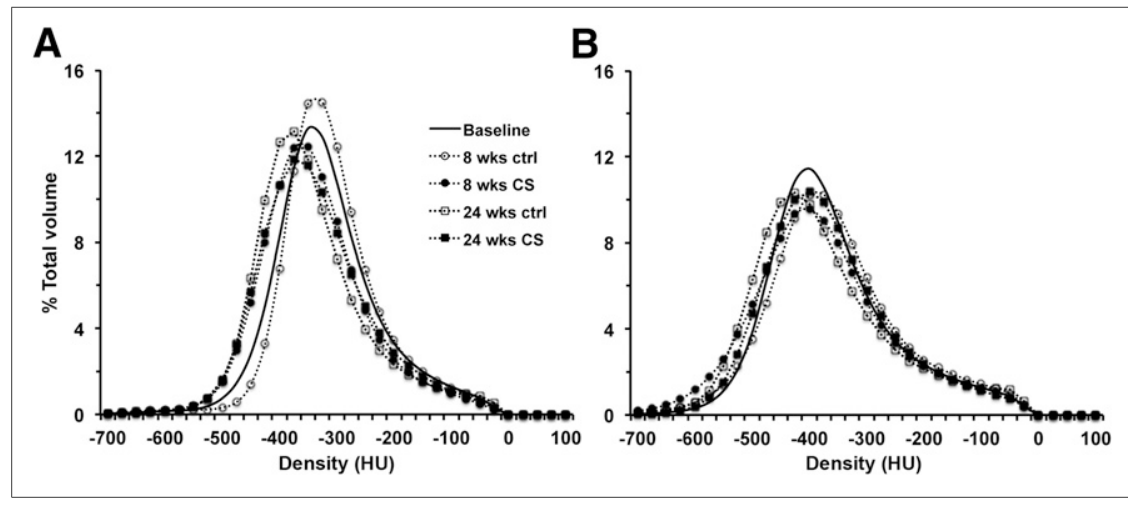

FIGURE 6. Respiration-gated CT densitometry. HU density distributions in percentage of total volume are shown for end-expiratory (A) and end-inspiratory (B) breathing states. Baseline, 8-wk control, 8-wk CS-exposed, 24-wk control, and 24-wk CS-exposed are shown. ctrl $=$ control. 
ence was found between these age-matched groups. Airspace enlargement, as observed in control animals through both CT and histology, was potentially involved in the increase in V/Q heterogeneity, as measured by the $\mathrm{SD}$ of the $\log (\mathrm{V} / \mathrm{Q})$, because these results increased with aging in the absence of aberrant immunologic processes $(16,27)$.

In addition to airspace enlargement and inflammation, other pathologies associated with COPD likely contribute to V/Q mismatching seen clinically. Inflammatory exudate, small-airway remodeling, increased mucus production, and loss of tethering forces could lead to an altered ventilation profile through the closure of small airways. Although inflammation and airspace enlargement likely have a role in lung dysfunction, the levels of these pathologies observed in this study may not adequately account for all of the V/Q mismatching detected. A healthy pulmonary vasculature could adapt blood flow, predominantly through hypoxic vasoconstriction, to provide matched V/Q (28). This system is impaired in COPD patients (29), and vascular abnormalities have been identified in other models of CS exposure in small animals $(30,31)$.

Although murine models may not reflect all of the pathologies associated with COPD at once, they allow the study of individual aspects of disease. Our results indicate that inflammation is likely responsible for some of the V/Q abnormalities observed and that airspace enlargement may have a limited role in this model. Further work in several models of COPD pathologies will be necessary to determine the relative contributions of these pathologies to V/Q mismatching. Because smoking cessation has been shown to reduce some inflammatory parameters (32), the study of smoking cessation in this controlled system will be a necessary step toward confirming the roles of the pathologies observed toward V/Q mismatching and toward demonstrating the ability of this methodology to investigate therapeutic approaches.

Through a per-voxel analysis of V/Q, this study has demonstrated that chronic exposure to the noxious and particulate components of CS is enough to elicit alterations in gas exchange capacity. V/Q mismatching is a sensitive, noninvasive measure of lung dysfunction that can provide insight into many of the pathologies and obstructions that cause COPD. In COPD, and preclinical models thereof, the ability to detect incipient disease is necessary to allow for the investigation of the underlying biologic mechanisms. Further, the ability to track progression provides a system in which interventions can be tested. Within this CS exposure model, V/Q SPECT has proven useful to establish both detection and progression and can be used further in the investigation of treatment options.

\section{DISCLOSURE}

The costs of publication of this article were defrayed in part by the payment of page charges. Therefore, and solely to indicate this fact, this article is hereby marked "advertisement" in accordance with 18 USC section 1734. Tuition support for this study was provided by Firestone Institute for Respiratory Health (Hamilton, Ontario, Canada). This study was supported in part by a Firestone Institute of Respiratory Health-AstraZeneca Collaboration unrestricted grant and the Canadian Institutes of Health Research. N. Renée Labiris holds an internal Department of Medicine Career Award. No other potential conflict of interest relevant to this article was reported.

\section{ACKNOWLEDGMENTS}

We thank Dr. Troy H. Farncombe and Chantal Saab for their expertise and contributions toward imaging and Joanna Kasinska for expert technical support, as well as Dr. Karen Gulenchyn and the McMaster Department of Nuclear Medicine, Hamilton Health Sciences (Hamilton, Ontario, Canada) for use of the Technegas system.

\section{REFERENCES}

1. Rabe KF, Hurd S, Anzueto A, et al. Global strategy for the diagnosis, management, and prevention of chronic obstructive pulmonary disease: GOLD executive summary. Am J Respir Crit Care Med. 2007;176:532-555.

2. Lopez AD, Shibuya K, Rao C, et al. Chronic obstructive pulmonary disease: current burden and future projections. Eur Respir J. 2006;27:397-412.

3. Barnes PJ. Immunology of asthma and chronic obstructive pulmonary disease. Nat Rev Immunol. 2008;8:183-192.

4. Hogg JC. Pathophysiology of airflow limitation in chronic obstructive pulmonary disease. Lancet. 2004;364:709-721.

5. Han MK, Agusti A, Calverley PM, et al. Chronic obstructive pulmonary disease phenotypes: the future of COPD. Am J Respir Crit Care Med. 2010;182:598604.

6. Hogg JC, Timens W. The pathology of chronic obstructive pulmonary disease. Annu Rev Pathol. 2009;4:435-459.

7. Yoon HI, Sin DD. Biomarkers of therapeutic response in patients with chronic obstructive pulmonary disease: a critical review of the literature. Drugs. 2011;71:1821-1837.

8. Rabe KF, Wedzicha JA. Controversies in treatment of chronic obstructive pulmonary disease. Lancet. 2011;378:1038-1047.

9. Rodríguez-Roisin R, Drakulovic M, Rodriguez DA, Roca J, Barbera JA, Wagner PD. Ventilation-perfusion imbalance and chronic obstructive pulmonary disease staging severity. J Appl Physiol. 2009;106:1902-1908.

10. Wagner PD. The multiple inert gas elimination technique (MIGET). Intensive Care Med. 2008;34:994-1001.

11. Roach PJ, Bailey DL, Harris BE. Enhancing lung scintigraphy with singlephoton emission computed tomography. Semin Nucl Med. 2008;38:441449.

12. Petersson J, Sanchez-Crespo A, Rohdin M, et al. Physiological evaluation of a new quantitative SPECT method measuring regional ventilation and perfusion. J Appl Physiol. 2004;96:1127-1136.

13. Jögi J, Ekberg M, Jonson B, Bozovic G, Bajc M. Ventilation/perfusion SPECT in chronic obstructive pulmonary disease: an evaluation by reference to symptoms, spirometric lung function and emphysema, as assessed with HRCT. Eur J Nucl Med Mol Imaging. 2011;38:1344-1352.

14. Suga K, Kawakami Y, Koike H, et al. Lung ventilation-perfusion imbalance in pulmonary emphysema: assessment with automated V/Q quotient SPECT. Ann Nucl Med. 2010;24:269-277.

15. Stevenson CS, Birrell MA. Moving towards a new generation of animal models for asthma and COPD with improved clinical relevance. Pharmacol Ther. 2011;130:93-105.

16. Jobse BN, Rhem RG, McCurry CA, Wang IQ, Labiris NR. Imaging lung function in mice using SPECT/CT and per-voxel analysis. PLOS ONE. 2012;7: e42187.

17. Botelho FM, Gaschler GJ, Kianpour S, et al. Innate immune processes are sufficient for driving cigarette smoke-induced inflammation in mice. Am J Respir Cell Mol Biol. 2010;42:394-403.

18. Farncombe TH. Software-based respiratory gating for small animal conebeam CT. Med Phys. 2008;35:1785-1792. 
19. Jobse BN, Johnson JR, Farncombe TH, et al. Evaluation of allergic lung inflammation by computed tomography in a rat model in vivo. Eur Respir J. 2009;33:1437-1447.

20. Froese AR, Ask K, Labiris R, et al. Three-dimensional computed tomography imaging in an animal model of emphysema. Eur Respir J. 2007;30:10821089.

21. Mariani G, Bruselli L, Kuwert T, et al. A review on the clinical uses of SPECT/ CT. Eur J Nucl Med Mol Imaging. 2010;37:1959-1985.

22. Bajc M, Neilly JB, Miniati M, et al. EANM guidelines for ventilation/perfusion scintigraphy: Part 1. Pulmonary imaging with ventilation/perfusion single photon emission tomography. Eur J Nucl Med Mol Imaging. 2009;36:13561370 .

23. King GG, Harris B, Mahadev S. V/Q SPECT: utility for investigation of pulmonary physiology. Semin Nucl Med. 2010;40:467-473.

24. Wagner PD, Dantzker DR, Dueck R, Clausen JL, West JB. Ventilation-perfusion inequality in chronic obstructive pulmonary disease. J Clin Invest. 1977;59:203216.

25. Barbera JA, Ramirez J, Roca J, Wagner PD, Sanchez-Lloret J, RodriguezRoisin R. Lung structure and gas exchange in mild chronic obstructive pulmonary disease. Am Rev Respir Dis. 1990;141:895-901.
26. Schroeder T, Vidal Melo MF, Musch G, Harris RS, Winkler T, Venegas JG. PET imaging of regional ${ }^{18} \mathrm{~F}-\mathrm{FDG}$ uptake and lung function after cigarette smoke inhalation. J Nucl Med. 2007;48:413-419.

27. Sprung J, Gajic O, Warner DO. Review article: age related alterations in respiratory function - anesthetic considerations. Can J Anaesth. 2006;53:1244-1257.

28. Glenny RW. Determinants of regional ventilation and blood flow in the lung. Intensive Care Med. 2009;35:1833-1842.

29. Vidal Melo MF, Winkler T, Harris RS, Musch G, Greene RE, Venegas JG. Spatial heterogeneity of lung perfusion assessed with ${ }^{13} \mathrm{~N}$ PET as a vascular biomarker in chronic obstructive pulmonary disease. J Nucl Med. 2010;51:57-65.

30. Wright JL, Churg A. Short-term exposure to cigarette smoke induces endothelial dysfunction in small intrapulmonary arteries: analysis using guinea pig precision cut lung slices. J Appl Physiol. 2008;104:1462-1469.

31. Ferrer E, Peinado VI, Diez M, et al. Effects of cigarette smoke on endothelial function of pulmonary arteries in the guinea pig. Respir Res. 2009;10: 76.

32. Wen Y, Reid DW, Zhang D, Ward C, Wood-Baker R, Walters EH. Assessment of airway inflammation using sputum, BAL, and endobronchial biopsies in current and ex-smokers with established COPD. Int J Chron Obstruct Pulmon Dis. 2010;5:327-334. 